\title{
Changes of Electrocardiogram and Myocardial Enzymes in Patients with Intracerebral Hemorrhage
}

\author{
Guannan Qin, ${ }^{1,2}$ Chuanyang Dai, ${ }^{2}$ Shuang Feng, ${ }^{3}$ and Guofeng Wu $\mathbb{D}^{2}$ \\ ${ }^{1}$ Guizhou Medical University, Guian New Area University Town, Guizhou Province 550025, China \\ ${ }^{2}$ Emergency Department, Guizhou Province, The Affiliated Hospital of Guizhou Medical University, No. 28, Guiyijie Road, \\ Liuguangmen, Guiyang City 550004, China \\ ${ }^{3}$ Emergency Department, Shandong Province, Zibo Central Hospital, No. 54, Gongqingtuan West Road, Zhangdian District, \\ Zibo City 255020, China
}

Correspondence should be addressed to Guofeng Wu; wuguofeng3013@sina.com

Received 22 December 2021; Revised 17 January 2022; Accepted 18 January 2022; Published 1 February 2022

Academic Editor: XIANWEI ZENG

Copyright (C) 2022 Guannan Qin et al. This is an open access article distributed under the Creative Commons Attribution License, which permits unrestricted use, distribution, and reproduction in any medium, provided the original work is properly cited.

Purpose. Cardiac complications are common in patients with spontaneous intracerebral hemorrhage (ICH). The present study is aimed at observing the incidence of cardiac complications after ICH, so as at improving the understanding of the relationship between cardiac complications and ICH. Methods. This is a retrospective study on analyzing electrocardiogram (ECG) and serum myocardial enzyme of 208 patients with ICH admitted to a tertiary hospital from 2018 to 2019. For each patient, demographics, medical history, clinical presentation, ECG, serum myocardial enzyme, and head CT on admission were reviewed. Mortality was noted. Results. Among the 208 patients, 145 (69.71\%) had one or more ECG abnormalities. The top three abnormalities were corrected QT interval (QTc) prolongation 52 (25\%), ST depression 48 (23.08\%), and T wave inversion $38(18.27 \%)$. One hundred and thirty-nine patients $(66.83 \%)$ had increased serum levels of at least one kind of myocardial enzyme, which were high-sensitive cardiac troponin T (hs-cTnT) 79 (37.98\%), lactic dehydrogenase (LDH) 80 (38.46\%), creatine kinase (CK) 57 (27.40\%), and creatine kinase-myocardial subfraction (CKMB) 57 (27.40\%). The logistic regression analysis showed the following: secondary intraventricular hemorrhage (SIVH) (odds ratio (OR) 5.32; 95\% confidence interval (CI) 2.55-11.08; $p<0.001)$ and hematoma volume $>30 \mathrm{ml}$ (OR $3.81 ; 95 \%$ CI $1.86-7.81 ; p<0.001)$ were independent predictive factors of QTc prolongation; thalamus location (OR 5.79; 95\% CI 1.94-17.28; $p<0.05$ ), hematoma volume $>30 \mathrm{ml}$ (OR 24.187; 95\% CI 3.14-186.33; $p<0.05$ ), insular involvement (OR 19.08; 95\% CI 5.77-63.07; $p<0.001$ ), and SIVH (OR 2.62; 95\% CI 1.69-5.86; $p<0.05)$ were independent predictive factors of ST depression; insular involvement (OR 2.90; 95\% CI 1.12-7.50; $p<0.05)$ and hematoma volume $>30 \mathrm{ml}(\mathrm{OR} 1.98 ; 95 \%$ CI 1.06-3.70; $p<0.05)$ were independent predictive factors of increase of CK; Glasgow Coma Scale (GCS) (OR 0.86; 95\% CI 0.78-0.98; $p<0.05$ ) and insular involvement (OR 5.56; 95\% CI 1.98-15.62; $p<0.05$ ) were independent predictive factors of increase of CKMB; SIVH (OR 2.05; 95\% CI 1.07-3.92; $p<0.05$ ) was independent predictive factor of increase of LDH; age (OR 1.03; 95\% CI 1.01-1.06; $p<0.05$ ), blood glucose on admission (OR 1.10; 95\% CI 1.01-1.20; $p<0.05$ ), and history of antiplatelet drug use (OR 3.50; $95 \%$ CI 1.0112.12; $p<0.05)$ were independent predictive factors of hs-cTnT. All the injury indexes were not related to in-hospital mortality. Conclusion. The study suggests that insular involvement, hematoma volume $>30 \mathrm{ml}$, and SIVH are the strongest risk factors for ECG abnormalities and elevated myocardial enzymes after ICH followed which are the risk factors such as GCS, age, admission blood glucose, and ICH location in the thalamus. 


\section{Introduction}

Despite advances in stroke care management, spontaneous intracerebral hemorrhage (ICH) is still a kind of serious stroke with high morbidity and mortality [1]. Secondary systemic complications are common and largely affect the outcomes of patients with ICH [2]. It is extremely important to identify and manage complications associated with clinical outcomes after ICH.

In fact, cardiac complications are frequently found in patients with acute stroke. Relevant studies mainly focused on ischemic stroke and subarachnoid hemorrhage, but few studies are on ICH $[3,4]$. A study has shown that cardiac complications may occur in $1 \%$ to $4 \%$ of patients with ICH [5]. Markers of myocardial injury in the complications, such as myocardial markers and electrocardiogram (ECG), are associated with prognosis in patients with ICH $[6,7]$. It is reported that ECG abnormalities after ICH are very common (56\%-81\%) $[8,9]$, which includes prolonged corrected QT (QTc) interval, ST-T changes, arrhythmias, and atrioventricular nodal blocks [10]. The most common cardiac biomarker in patients with ICH is troponin I [11]. The 2015 American Heart Association/American Stroke Association ICH guidelines recommend cardiac workup of patients with spontaneous $\mathrm{ICH}$, including both ECG and cardiac troponin, to assess active coronary ischemia and concomitant myocardial injury [12].

However, little is known about the relationship between these markers and various characteristics of ICH [13]. Therefore, we investigated the prevalence of myocardial injury in patients with ICH and the possible associations with clinical and radiological findings. Our study aimed at improving the understanding of the possible association between myocardial injury and clinical characteristics in patients with ICH. Furthermore, it could be also helpful to identify high-risk patients and predict possible adverse events.

\section{Materials and Methods}

2.1. Patients. Ethics approval was obtained from the local institutional review board (NO. S2017-02). Because it was a retrospective study, no written informed consent was required. Between 2018 and 2019, 274 patients with spontaneous ICH were admitted to the Emergency Neurology Department, Guizhou Medical University. General inclusion criteria contained spontaneous ICH confirmed by computed tomography (CT) scan, with the age being older than 18 years. Exclusion criteria were CT angiography (CTA) or magnetic resonance angiography (MRA) diagnosis of ICH due to brain tumor or vascular abnormalities, insufficient clinical data, ECG recordings, and serum myocardial enzyme on admission. Sixty-six patients were excluded due to brain tumor $(N=4)$, vascular abnormalities $(N=8)$, insufficient clinical data $(N=22)$, and lack of ECG recordings or serum myocardial enzyme $(N=32)$. Therefore, the subjects of the study included 208 patients in total (Figure 1).

2.2. Data Collection. The data were retrospectively analyzed to determine such clinical characteristics of the patients as age, sex, medical history, time of onset at admission, Glasgow
Coma Scale (GCS), location of hematoma, side of hematoma, presence of insular involvement, secondary intraventricular hemorrhage (SIVH), hematoma volume, blood tests (hemoglobin, platelet, white cell, and coagulation level), ECG findings, and level of serum myocardial enzyme. The location of hematoma was classified into basal ganglia, lobar, thalamus, cerebellum, brain stem, and primary intraventricular hemorrhage $(\mathrm{PIVH})$. Hematoma volume was measured through the $\mathrm{ABC} / 2$ method. Within 1 hour after admission, laboratory tests (blood tests, ECG, etc.) were performed for all involved patients. Mortality during hospitalization was reviewed.

2.3. ECG Analyses. ECG (12-lead) recordings were collected on admission before treatment at a paper speed of 25 millimeters per second, with an amplitude calibration of 10 millimeters per millivolt, and were analyzed by the same attending cardiologist, who did not know the details of the patients. Abnormalities of ECG are judged by the following criteria [6]: (a) rhythm: sinus rhythm, atrial fibrillation, or ectopic beats; (b) heart rate: sinus bradycardia ( $<60$ beats/minute) and sinus tachycardia ( $>100$ beats/min); (c) PR interval: short $<0.12$ seconds and prolonged $>0.2$ seconds; (d) QRS complex width: prolonged $>0.12$ seconds; (e) corrected QT interval (QTc) (using the Bazett formula): prolonged at $>0.45$ seconds in females and $>0.44$ seconds in males; (f) ST segment depression: downsloping or horizontal $>0.05$ millivolts; (g) ST segment elevation: convexity of the ST segment upward $>$ 0.1 millivolts; and (h) $\mathrm{T}$ wave inversion: negative $\mathrm{T}$ wave of $\geq 1$ millimeters in depth in two or more contiguous leads, with exclusion of leads aVR, III, and V1.

2.4. The Analysis of Serum Myocardial Enzyme. The elevation standard of myocardial enzyme is judged according to the reference range of our hospital's laboratory: (a) high creatine kinase (CK) value: >198 U/l; (b) high creatine kinasemyocardial subfraction $(\mathrm{CKMB})$ value: $>25 \mathrm{U} / \mathrm{l}$; (c) high lactic dehydrogenase $(\mathrm{LDH})$ value: $>250 \mathrm{U} /$; and (d) high-sensitive cardiac troponin T (hs-cTnT) value: $>0.014 \mathrm{ng} / \mathrm{ml}$.

2.5. Other Blood Tests. The standards for these tests also followed the hospital's laboratory criteria: (a) leukocytosis: white blood cell count $>10 \times 10^{9} / \mathrm{l}$; (b) low platelet count: platelet count $<100 \times 10^{9} / 1$; (c) abnormal hemoglobin level: hemoglobin levels up or down; and (d) coagulation abnormalities: one or more of the terms of prothrombin time (PT), activated partial thromboplastin time (APTT), international normalized ratio (INR), and D-dimer and fibrinogen up or down.

2.6. Statistical Analysis. The descriptive statistics are expressed as the mean \pm standard deviation (SD) or medians with interquartile ranges (IQR) for the continuous variables, while the categorical variables are expressed as percentages, as appropriate. Univariate analysis was used to determine the possible relationships between each ECG abnormality and clinical features, as well as each elevated serum myocardial enzyme and clinical features, by means of either the chi-square test or Fisher's exact test. If an ECG abnormality was observed in more than $10 \%$ of patients, it was analyzed. 


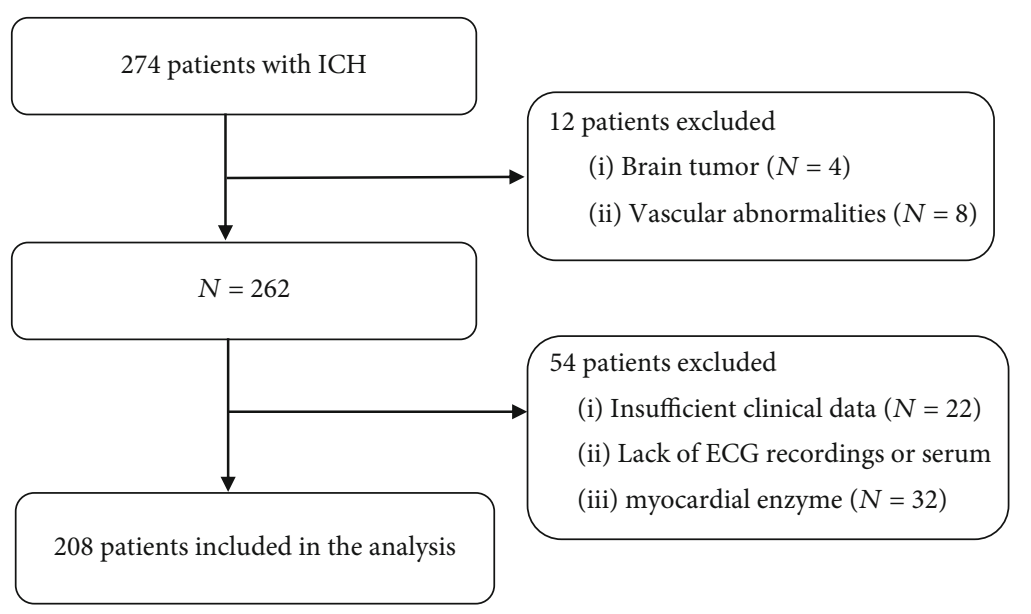

Figure 1: Research flow chart.

The relationship between in-hospital mortality and markers of cardiac injury, including ECG abnormalities observed in more than $10 \%$ of patients and serum myocardial enzymes, was also analyzed using the methods mentioned above.

The analysis of multivariate logistic regression was used to determine the independent correlation factors with ECG abnormalities and elevated serum myocardial enzymes and mortality. The variables with a significant level of $p<0.05$ in the univariate analysis were included as independent variables in the logistic regression analyses. Only those variables with $p<0.05$ in two-tailed tests were retained within the model. Odds ratios (OR) and 95\% confidence intervals (CI) were reported. A value of $p<0.05$ was regarded as statistical significance. All statistical analysis was conducted by virtue of SPSS (version 22.0; IBM Corporation, Armonk, NY, USA).

\section{Results}

The results of the clinical information, level of myocardial enzyme, and imaging findings of 208 patients with ICH are summarized in Table 1 . There were 136 males (65.38\%) and 72 females, with a mean age of 61.23 years (range: 29-93). The GCS was $\leq 8$ in 60 cases $(28.85 \%)$. Past history included hypertension in 140 cases (67.31\%), type 2 diabetes in 16 (7.69\%), cerebrovascular diseases in $32(15.38 \%)$, coronary heart disease in 11 (5.29\%), smoking history in 76 (36.54\%), drinking history in 45 (21.63\%), history of antiplatelet drug use in $14(6.73 \%)$, and anticoagulant drug use in $1(0.48 \%)$. The mean time of onset on admission was 6 hours $(3,24)$. The mean blood glucose was $7.43 \mathrm{mmol} / \mathrm{l}(5.93,9.97)$ on admission. Hematoma was located in the basal ganglia in 100 cases $(48.08 \%)$, lobar region in $62(29.81 \%)$, thalamus in 28 (13.46\%), cerebellum in $11(5.29 \%)$, brain stem in $4(1.92 \%)$, and primary intraventricular hemorrhage in $1(0.48 \%)$. Hematoma involved the insular lobe in 20 cases $(9.62 \%)$ and secondary intraventricular hemorrhage (26.92\%) in 56 cases. 96 cases $(46.15 \%)$ had hematoma on the right side. The hematoma was $>30 \mathrm{ml}$ in 87 cases (41.83\%), and the mean volume was $27 \mathrm{ml}$ $(10,50)$. The blood routine tests showed leukocytosis in 8 cases (3.85\%), low platelet count in 2 cases $(0.96 \%)$, abnormal hemoglobin level in 3 cases (1.44\%), and coagulation abnormalities in 2 cases $(0.96 \%)$. One or more ECG abnormalities were observed in 145 cases (69.71\%). The most common ECG abnormality was QTc prolongation (25.00\%), followed by ST segment depression (23.08\%) and $\mathrm{T}$ wave inversion (18.27\%). Elevated levels of one more kind of abnormal myocardial enzymes were observed in 139 patients (66.83\%). The most common type was LDH (38.4\%), followed by hs-cTnT (37.9\%), CK 57 (27.40\%), and CKMB 57 (27.40\%). Relevant ECG abnormalities that reached at least $10 \%$ of prevalence in the cohort were included in a univariate analysis and are illustrated in Tables 2 and 3. Relevant elevated myocardial enzymes were included in a univariate analysis and are illustrated in Tables 2 and 4 .

3.1. Analysis of QTc Prolongation. The univariate analysis presented that QTc prolongation was associated with location of the cerebellum $(p=0.049)$, SIVH $(p<0.001)$, and hematoma volume $>30 \mathrm{ml}(p<0.001)$. The logistic regression analysis showed that SIVH (OR 5.32; 95\% CI 2.5511.08; $p<0.001$ ) and hematoma volume $>30 \mathrm{ml}$ (OR 3.81; 95\% CI 1.86-7.81; $p<0.001)$ were independent predictive factors of QTc prolongation.

3.2. Analysis of ST Depression. The univariate analysis showed that ST depression was associated with GCS $\leq 8 \quad(p=0.009)$, location of the thalamus $(p=0.029)$, insular involvement $(p<0.001)$, SIVH $(p=0.009)$, and hematoma volume $>30 \mathrm{ml}$ $(p=0.008)$. The logistic regression analysis depicts that location of the thalamus (OR 5.79; 95\% CI 1.94-17.28; $p<0.05$ ), hematoma volume $>30 \mathrm{ml}$ (OR 3.68; 95\% CI 1.40-9.67; $p<$ 0.05), insular involvement (OR 19.08; 95\% CI 5.77-63.07; $p$ $<0.001$ ), and SIVH (OR 2.62; 95\% CI 1.17-5.86; $p<0.05$ ) were independent predictive factors of ST depression.

3.3. Analysis of Inverted $T$ Wave. The univariate analysis showed that inverted $\mathrm{T}$ wave was associated with age $(p=0.006)$, history of cerebrovascular diseases $(p=0.01)$, history of antiplatelet drug use $(p=0.001)$, location of basal ganglia $(p<0.001)$, lobar $(p<0.001)$, and thalamus $(p<0.001)$. The logistic regression analysis showed that there was no independent predictive factor of inverted $\mathrm{T}$ wave. 
TABLE 1: Baseline characteristics of 208 patients with ICH.

\begin{tabular}{|c|c|}
\hline Characteristics & Patient data \\
\hline \multicolumn{2}{|l|}{ Age } \\
\hline Years (SD) & $61.23(13.64)$ \\
\hline$\leq 60$ years, $n(\%)$ & $110(52.88)$ \\
\hline Male, $n(\%)$ & $136(65.38)$ \\
\hline \multicolumn{2}{|l|}{ History, $n(\%)$} \\
\hline History of hypertension & $140(67.31)$ \\
\hline History of type 2 diabetes & $16(7.69)$ \\
\hline History of cerebrovascular diseases & $32(15.38)$ \\
\hline History of coronary heart disease & $11(5.29)$ \\
\hline History of smoking & $76(36.54)$ \\
\hline History of drinking & $45(21.63)$ \\
\hline Antiplatelet drugs & $14(6.73)$ \\
\hline Anticoagulant drugs & $1(0.48)$ \\
\hline Time, h (IQR) & $6(21)$ \\
\hline $\mathrm{GCS} \leq 8, n(\%)$ & $60(28.85)$ \\
\hline Blood glucose, mmol/l (IQR) & $7.43(4.04)$ \\
\hline \multicolumn{2}{|l|}{ Location of hematoma, $n(\%)$} \\
\hline Basal ganglia & $100(48.08)$ \\
\hline Lobar & $62(29.81)$ \\
\hline Thalamus & $28(13.46)$ \\
\hline Cerebellum & $11(5.29)$ \\
\hline Brain stem & $4(1.92)$ \\
\hline Primary intraventricular hemorrhage & $1(0.48)$ \\
\hline Right-sided hematoma, $n(\%)$ & $96(46.15)$ \\
\hline Insular involvement, $n(\%)$ & $20(9.62)$ \\
\hline Secondary intraventricular hemorrhage, $n(\%)$ & $56(26.92)$ \\
\hline \multicolumn{2}{|l|}{ Hematoma volume } \\
\hline Median, ml (IQR) & $27(40)$ \\
\hline$>30 \mathrm{ml}, n(\%)$ & $87(41.83)$ \\
\hline \multicolumn{2}{|l|}{ Other blood tests, $n(\%)$} \\
\hline Leukocytosis & $8(3.85)$ \\
\hline Low platelet count & $2(0.96)$ \\
\hline Abnormal hemoglobin level & $3(1.44)$ \\
\hline Coagulation abnormalities & $2(0.96)$ \\
\hline \multicolumn{2}{|l|}{ ECG abnormalities, $n(\%)$} \\
\hline QTc prolongation & $52(25.00)$ \\
\hline ST depression & $48(23.08)$ \\
\hline Inverted $\mathrm{T}$ wave & $38(18.27)$ \\
\hline LBBB & $11(5.29)$ \\
\hline PVC & $8(3.85)$ \\
\hline $\mathrm{RBBB}$ & $3(1.44)$ \\
\hline Atrial fibrillation & $2(0.96)$ \\
\hline \multicolumn{2}{|l|}{ Myocardial enzymes values (IQR) } \\
\hline $\mathrm{CK}, \mathrm{U} / \mathrm{l}$ & $122.25(129.05)$ \\
\hline CKMB, U/l & $19.4(12.14)$ \\
\hline $\mathrm{LDH}, \mathrm{U} / \mathrm{l}$ & $233(84.5)$ \\
\hline hs-cTnT, ng/ml & $0.012(0.011)$ \\
\hline Elevated myocardial enzymes, $n(\%)$ & \\
\hline
\end{tabular}

TABLE 1: Continued.

\begin{tabular}{cc}
\hline Characteristics & Patient data \\
\hline CK & $57(27.4)$ \\
CKMB & $57(27.4)$ \\
LDH & $80(38.46)$ \\
hs-cTnT & $79(37.98)$ \\
Mortality, $n(\%)$ & $17(8.17)$ \\
\hline
\end{tabular}

ICH: intracerebral hemorrhage; SD: standard deviation; Time: time of onset on admission; GCS: Glasgow Coma Scale; ECG: electrocardiogram; QTc: corrected QT interval; LBBB: left bundle branch block; PVC: premature ventricular contraction; RBBB: right bundle branch block; CK: creatine kinase; CKMB: creatine kinase-myocardial subfraction; LDH: lactic dehydrogenase; hs-cTnT: high-sensitive cardiac troponin $\mathrm{T}$.

3.4. Analysis of $\angle B B B$. The univariate analysis showed that LBBB was associated with GCS $(p=0.003)$, primary intraventricular hemorrhage $(p=0.046)$, hematoma volume $>30$ $\mathrm{ml}(p=0.001)$, and abnormal hemoglobin level $(p<0.001)$. The logistic regression analysis showed that there was no independent predictive factor of LBBB.

3.5. Analysis of Increase in CK. The univariate analysis showed that the increase in $\mathrm{CK}$ was associated with insular involvement $(p=0.017)$ and hematoma volume $>30 \mathrm{ml}$ $(p=0.024)$. The logistic regression analysis showed that insular involvement (OR 2.9; 95\% CI 1.12-7.50; $p<0.05$ ) and hematoma volume $>30 \mathrm{ml}$ (OR 1.98; 95\% CI 1.06$3.70 ; p<0.05)$ were independent predictive factors of the increase in $\mathrm{CK}$.

3.6. Analysis of Increase in $C K M B$. The univariate analysis showed that the increase in $\mathrm{CKMB}$ was associated with time of onset at admission $(p=0.039)$, GCS $(p<0.001)$, blood glucose $(p=0.02)$, and insular involvement $(p<0.001)$. The logistic regression analysis showed that GCS (OR 0.87; 95\% CI $0.79-0.95 ; p<0.05$ ) and insular involvement (OR 5.57; 95\% CI 1.98-15.64; $p=0.001$ ) were independent predictive factors of the increase in CKMB.

3.7. Analysis of Increase in $L D H$. The univariate analysis showed that the increase in LDH was associated with GCS $(p=0.013)$, blood glucose $(p=0.008)$, SIVH $(p=0.007)$, and hematoma volume $>30 \mathrm{ml} \quad(p=0.006)$. The logistic regression analysis showed that SIVH (OR 2.05; 95\% CI $1.071-3.92 ; p<0.05)$ was an independent predictive factor of the increase in LDH.

3.8. Analysis of Increase in $h s-c T n T$. The univariate analysis showed that the increase in hs-cTnT was associated with age ( $p=0.003)$, history of T2D (type 2 diabetes) $(p=0.008)$, history of antiplatelet drug use $(p=0.007)$, blood glucose ( $p=0.024)$, location of basal ganglia $(p=0.046)$, and leukocytosis $(p=0.025)$. The analysis of logistic regression showed that age (OR 1.03; 95\% CI 1.01-1.06; $p<0.05$ ), blood glucose (OR 1.10; 95\% CI 1.01-1.20; $p<0.05$ ), and history of antiplatelet drug use (OR 3.504; 95\% CI 1.01-12.12; $p<0.05)$ were independent predictive factors of the increase in hs-cTnT. 


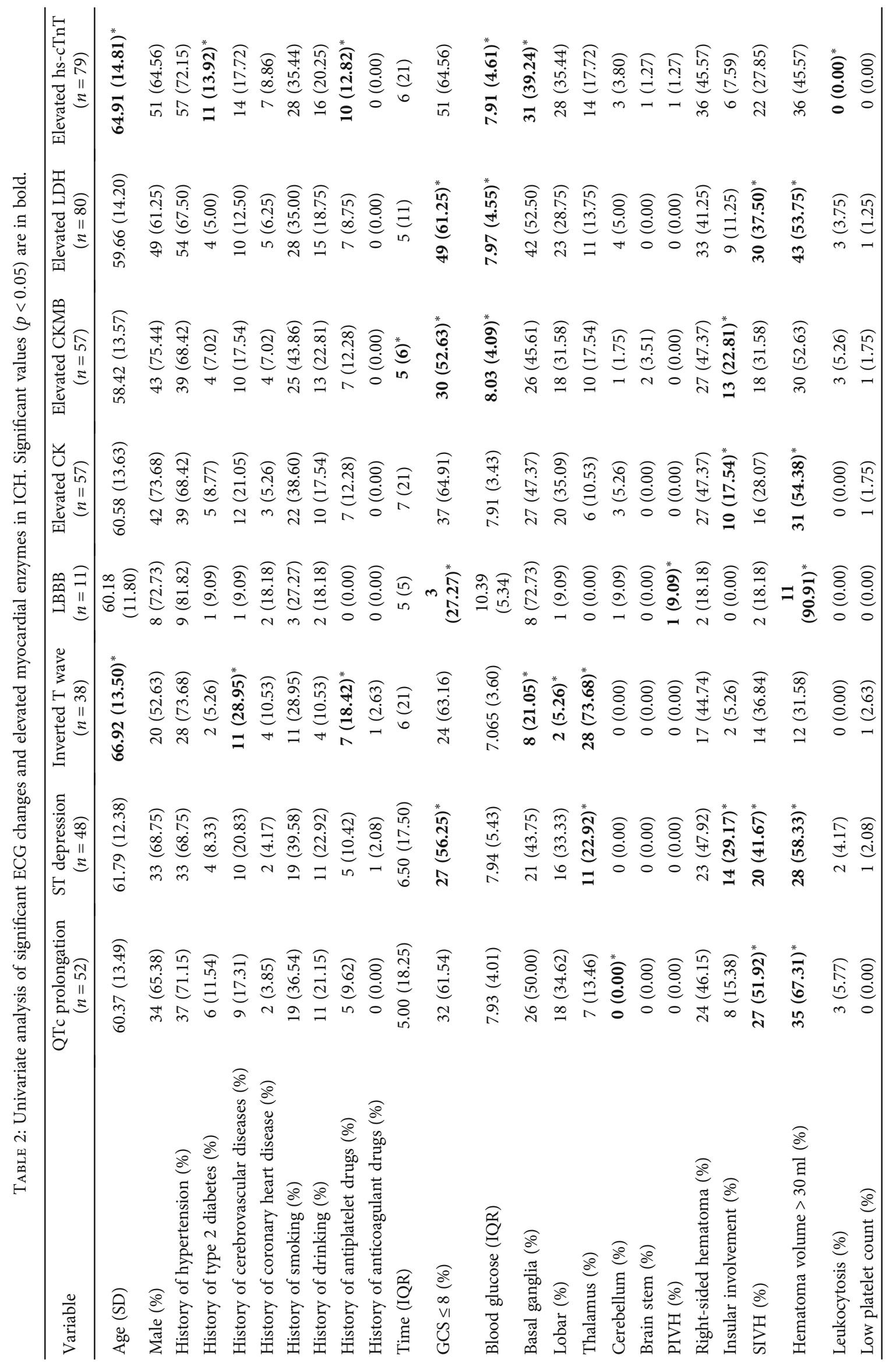




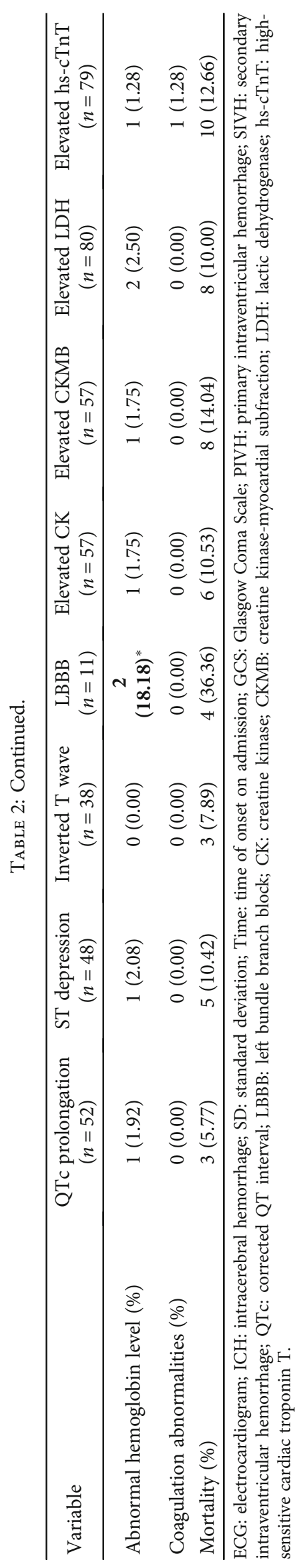




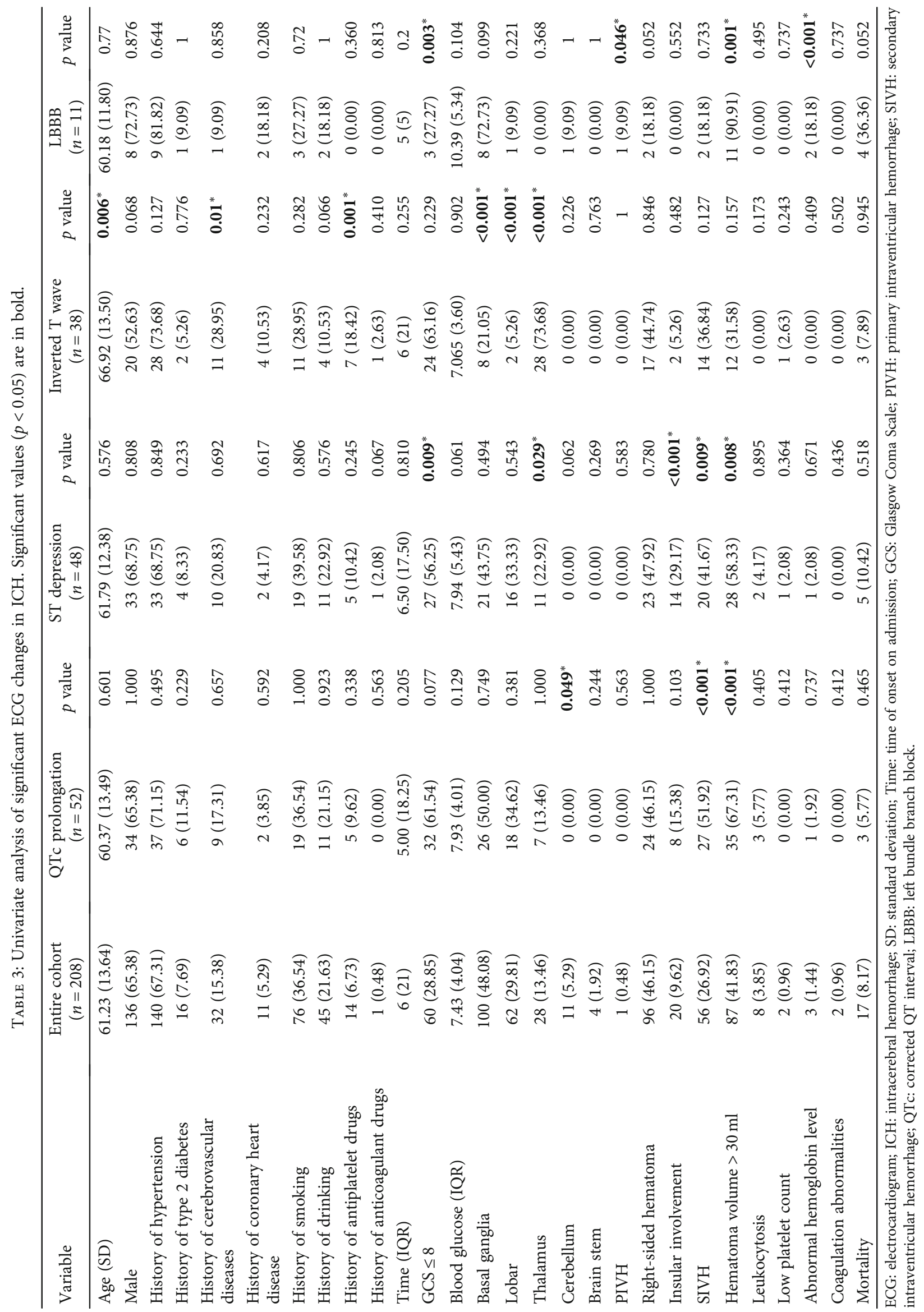




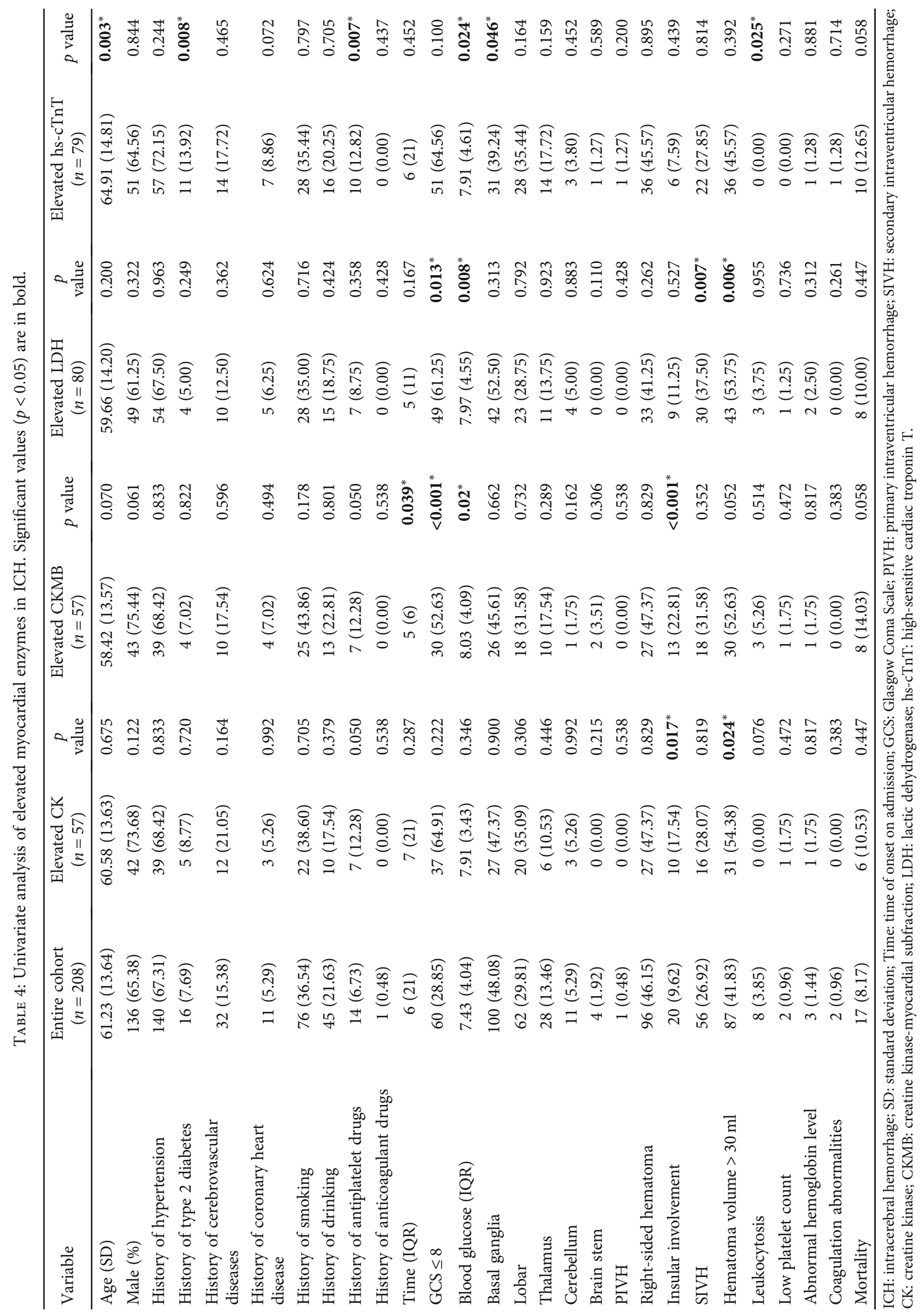


3.9. Impact of Cardiac Injury Markers on Mortality. In a univariate analysis, there was no ECG abnormality observed in more than $10 \%$ of patients and elevated serum myocardial enzyme associated with in-hospital mortality.

\section{Discussion}

Many researchers have observed the changes of ECG and myocardial enzymes in ICH patients. However, their studies only attach the importance to one or two markers of ECG and myocardial enzymes, the analysis on both of which is less researched in the same group of patients. Therefore, it is impossible to fully discover what factors affect the heart after ICH. In this study, ECG and myocardial enzymes of 208 patients with ICH were analyzed together so as to further clarify the relationship between different characteristics of ICH and cardiac complications. The result showed that insular involvement, hematoma volume $>30 \mathrm{ml}$, and SIVH were the strongest risk factors triggering the abnormalities of ECG and myocardial enzyme after ICH, followed by GCS, age, admission blood glucose, and location of the thalamus.

ECG abnormalities $(69.71 \%)$ were common in patients with ICH in our study, which was basically consistent with the incidence reported in literature $[8,9]$. The most common ECG abnormalities were prolonged QTC, ST segment depression, and inverted $\mathrm{T}$ wave. Some researchers have also found that these abnormalities were often observed during the acute phase of ICH [14]. Then, we examined the factors associated with ECG abnormalities. We found that hematoma volume and SIVH were the two strongest determinants of ECG abnormalities, which is consistent with the previous reports [15].

In the study, elevated myocardial enzymes (74.52\%) were also found in the plasma of patients with $\mathrm{ICH}$, among which, LDH was the most common one, followed by hscTnT. Studies have shown that LDH levels are increased in patients with central nervous system diseases such as cerebral infarction and hypoxic-ischemic encephalopathy [16, 17], as well as ICH [18]. The proportion of increased troponin in this study (37.98\%) is higher than that in other studies (27.4\%) [6] and (22\%) [19]. It seems to have to do with the inconsistent types of cardiac troponin in the two studies. hscTnT was studied in this study while cTnI or cTnT in others. We examined the risk factors associated with the four myocardial enzymes observed. Insular involvement was the main determinant of myocardial enzyme elevation, related to two kinds. In addition, GCS, hematoma volume, SIVH, age, and blood glucose levels were also associated with one item, respectively. This is consistent with many studies, in which myocardial enzyme correlated with markers of disease severity such as hematoma volume, NIHSS score, and intraventricular hemorrhage [20-22].

Insula is important to cardiac sympathetic output maintenance. ICH patients whose insula was involved had an increased risk of cardiac complications [23], which is consistent with our findings. It also showed that the risk was elevated when the right hemisphere was involved. Although there is evidence that insular cortex-mediated sympathetic control is hemispheric dominant [24], the experimental results are still inconsistent [25]. However, our results did not show a differ- ence in ECG or serum myocardial enzyme effects between left and right hemisphere hematoma. The reason may be that our study was a clinical retrospective study, and the results were derived from animal experiments. Therefore, the hematoma location identified in our study is not as accurate as in animal studies, which seems to influence judgments of hemispheric laterality.

Hematoma extension to the third and fourth ventricles may trigger destruction of the baroreflex receptors around the area [26], because of the abundant network structures controlling autonomic nerve function around the third and fourth ventricles, involving the paraventricular nucleus of the hypothalamus, periaqueductal gray matter, brain stem, and so on. Expansion of the intraventricular hematoma may lead to acute autonomic nervous system disorders and elevated circulating catecholamine levels, which could lead to cardiac complications [27]. In addition, the central control of the autonomic nervous system was thought to be located in the insular cortex, cingulate gyrus, amygdala, and hypothalamus [28]. Cardiovascular autonomic centers may also involve the extrainsular regions and their interconnecting fibers [29]. Therefore, the larger the hematoma, the more severe the pressure on the central control area, and the more severe the damage to the contact fibers. This also increases the risk of heart complications and explains why both hematoma volume $>30 \mathrm{ml}$ and SIVH were independent risk factors for ECG and myocardial enzyme abnormities.

Our study found that lower GCS was closely related to the increase of CKMB after ICH and also an independent predictor. However, in patients with traumatic brain injury (TBI), researchers did not find association between GCS and the development of cardiac dysfunction [29]. An explanation for the difference could be that the damage of hematoma in ICH to the brain tissue is limited, while it is diffused in TBI. This kind of diffuse lesion may mask the effect of the autonomic nerve center on the heart. The study confirmed that cardiac complications were more frequent with increasing grading of diffuse brain injury [30]. In addition, GCS reflects the functional integrity of the brain stem. And the brain stem, especially the caudal autonomic centers, is very important for the nervous influences to the heart. One study observed the heart rate variability (HRV) in patients in coma due to different diseases. HRV was found a progressive trend associated with deepening of coma, assessed by the GCS [31]. This is consistent with our findings, asserting that GCS could predict the occurrence of cardiac complications in central nervous system diseases.

We demonstrated that location of the thalamus is an independent risk factor for ST depression. This is related to the fact that the thalamus is a relay station for autonomous afferent impulses to the insular cortex [32] and is also involved in the autonomic nervous system pathway [33]. Racho et al. had also noted the importance of the thalamus in the regulation of the ANS, through the observation of Takotsubo cardiomyopathy after a thalamic stroke [34].

Elevated troponin is frequently detected in patients with stroke $[6,35]$. In this study, admission blood glucose level, age, and history of antiplatelet drug use were found to be risk factors for elevated troponin. Type 2 diabetes has been proven 
to have a significant association with elevated hs-cTnT levels in community-dwelling population; fasting blood glucose played a crucial role in this association [36]. Sharain et al. studied 830 patients and found that older age was also associated with elevated hs-cTnT [37]. We believe that admission blood glucose and age also affect the increase of troponin after $\mathrm{ICH}$. The main reason why blood glucose and age affect the increase of troponin after ICH may be that hyperglycemia and old age reduce the cardiac tolerance of patients and are also prone to cardiac complications such as myocardial ischemia or necrosis after ICH.

History of antiplatelet drug use means those patients suffered from severe atherosclerosis, such as coronary artery occlusion. Scheitz et al. [38] believed that troponin may be chronically elevated or acutely elevated in patients with stroke, which needs to be determined by further evaluation. Thus, in our study, troponin may be chronically elevated in the patients with a history of antiplatelet drugs. The cause may be the coronary factors.

The relationship between injury markers and in-hospital mortality was further investigated. Compared to the published literature, alteration of the injury markers in patients with ICH was not found to be associated with in-hospital mortality. The previous reports showed an association between ECG abnormalities and outcome. However, it is arguable which abnormality can predict the prognosis. Some studies have shown that QT interval could predict the mortality of ICH patients [7, 39]. But other researchers disagreed with this hypothesis. In their study, inversion of $\mathrm{T}$ waves was the only ECG aberrancy that is related to the clinical outcome [9]. The same situation also occurs in the relationship between myocardial enzymes and clinical prognosis. Some argued that elevation in cardiac troponin levels has been associated with in-hospital mortality [11, 40, 41], but other studies suggested the opposite case [42]. The possible reason for the inconsistency of the results is that multiple factors independent from ICH could affect ECG and myocardial enzymes, such as medicine, medical history, and structural cardiac pathologies. As these variables could not be controlled completely in all the current researches, any conclusions drawn from this topic are not fully convincing and precise. Our research is no exception. Therefore, it is still uncertain what injury markers could accurately predict the prognosis until a better research method is designed. Another reason of our failure in discovering the correlation between cardiac injury markers and prognosis may be the choice of endpoint observed in this study. The purpose of this study was to determine factors for ECG and increased myocardial enzyme after ICH, so we only analyzed inhospital mortality. If we were able to include outcomes at 3-6 months, we might have a different finding.

This study had important clinical implications. The ECG and myocardial enzymes of the same group of $\mathrm{ICH}$ patients at admission were observed. The possible independent predictors of the early cardiac injury markers were analyzed, albeit this study did not find the relationship between these markers and in-hospital mortality. Our findings are still conducive for clinicians to early identify those patients with $\mathrm{ICH}$ who are at high risk of secondary heart injury and intervene in advance. This may improve the prognosis of the patients, but further research is needed.

4.1. Limitations. The study is limited in respect of retrospective feature. The single-center study reflected the cardiac complications associated with ICH in the specific patients of Guiyang. Therefore, the results may not be promoted in different groups of patients. It also had a small sample size, which reduced the statistical power and increased the chance of type 2 errors. Additionally, the dynamic changes of ECG and cardiac enzymes were not included as parameter into our study, resulting in a lack of data on whether these changes were transient or permanent. Due to the lack of complete outcome measures for the patients such as mortality at 3 to 6 months and scores from the Glasgow Outcome Scale or Modified Rankin Scale, this resulted in possibly ambiguous associations between cardiac injury markers and prognosis. Despite the limitations, our findings allow for some useful clinical speculations.

\section{Conclusion}

We studied ECG abnormalities and changes of serum myocardial enzymes and the relationship between these markers of cardiac damage and the detailed characteristics of $\mathrm{ICH}$ in a series of patients and demonstrated that insular involvement, hematoma volume $>30 \mathrm{ml}$, and SIVH were the strongest risk factors for ECG and myocardial enzyme abnormalities after ICH. The risk factors are as follows: GCS, age, admission blood glucose, and location of the thalamus. Further prospective studies are needed to corroborate our findings.

\section{Data Availability}

Data are available to researchers on request for purposes of reproducing the results or replicating the procedure by directly contacting the corresponding author.

\section{Disclosure}

The funding body did not take part in the design of the study and collection, analysis, and interpretation of data and in writing the manuscript.

\section{Conflicts of Interest}

The authors report no potential conflicts of interest.

\section{Acknowledgments}

This research was supported by the Natural Science Foundation of China (81971126/H0906) and Guizhou Science and Technology Foundation (qiankehe zhicheng (2017) 2881).

\section{References}

[1] B. A. Gross, B. T. Jankowitz, and R. M. Friedlander, "Cerebral intraparenchymal Hemorrhage," JAMA The Journal of the American Medical Association, vol. 321, no. 13, pp. 12951303, 2019. 
[2] M. Stein, G. F. Hamann, B. Misselwitz, E. Uhl, M. Kolodziej, and M. H. Reinges, "In-hospital mortality and complication rates in surgically and conservatively treated patients with spontaneous intracerebral hemorrhage," World Neurosurgery, vol. 88, pp. 306-310, 2016.

[3] Y. W. Kuo, M. Lee, Y. C. Huang, and J. D. Lee, "Initial inhospital heart rate is associated with three-month functional outcomes after acute ischemic stroke," BMC Neurology, vol. 21, no. 1, p. 222, 2021.

[4] A. Kerro, T. Woods, and J. J. Chang, "Neurogenic stunned myocardium in subarachnoid hemorrhage," Journal of Critical Care, vol. 38, pp. 27-34, 2017.

[5] R. J. Koivunen, E. Haapaniemi, J. Satopää, M. Niemelä, T. Tatlisumak, and J. Putaala, "Medical acute complications of intracerebral hemorrhage in young adults," Stroke Research and Treatment, vol. 2015, Article ID 357696, 7 pages, 2015.

[6] Y. He, Q. Liu, J. Wang, D. W. Wang, H. Ding, and W. Wang, "Prognostic value of elevated cardiac troponin I in patients with intracerebral hemorrhage," Clinical Cardiology, vol. 43, no. 4, pp. 338-345, 2020.

[7] S. M. Z. Ziabari, N. Akhundzadeh, M. Shakiba, and P. Keshavarz, "The relationship between QT interval and intra-hospital mortality in patients with spontaneous intracranial hemorrhage," Advanced Journal of Emergency Medicine, vol. 4, no. 2, p. e25, 2020.

[8] S. Takeuchi, K. Nagatani, N. Otani, K. Wada, and K. Mori, "Electrocardiograph abnormalities in intracerebral hemorrhage," Journal of Clinical Neuroscience, vol. 22, no. 12, pp. 1959-1962, 2015.

[9] M. Bree, Y. Roos, I. Bilt et al., "Prevalence and characterization of ECG abnormalities after intracerebral hemorrhage," Neurocritical Care, vol. 12, no. 1, pp. 50-55, 2009.

[10] C. D'Amore, M. Paciaroni, G. Silvestrelli et al., "Severity of acute intracerebral haemorrhage, elderly age and atrial fibrillation: independent predictors of poor outcome at three months," European Journal of Internal Medicine, vol. 24, no. 4, pp. 310-313, 2013.

[11] M. C. Garrett, R. J. Komotar, R. M. Starke, D. Doshi, M. L. Otten, and E. S. Connolly, "Elevated troponin levels are predictive of mortality in surgical intracerebral hemorrhage patients," Neurocritical Care, vol. 12, no. 2, pp. 199-203, 2010.

[12] L. B. Morgenstern, J. H. Rd, C. Anderson et al., "Guidelines for the management of spontaneous intracerebral hemorrhage: a guideline for healthcare professionals from the American Heart Association/American Stroke Association," Stroke, vol. 46, no. 7, p. 2032, 2015.

[13] A. Lele, V. Lakireddy, S. Gorbachov, N. Chaikittisilpa, V. Krishnamoorthy, and M. S. Vavilala, "A narrative review of cardiovascular abnormalities after spontaneous intracerebral hemorrhage," Journal of Neurosurgical Anesthesiology, vol. 31, no. 2, pp. 199-211, 2019.

[14] Z. Chen, P. Venkat, D. Seyfried, M. Chopp, T. Yan, and J. Chen, "Brain-heart interaction cardiac complications after stroke," Circulation Research, vol. 121, no. 4, pp. 451-468, 2017.

[15] A. Loggini, A. Mansour, F. E. Ammar et al., "Inversion of T waves on admission is associated with mortality in spontaneous intracerebral hemorrhage," Journal of Stroke and Cerebrovascular Diseases, vol. 30, no. 6, article 105776, 2021.

[16] Y. Lampl, Y. Paniri, Y. Eshel, and I. Sarova-Pinhas, "Cerebrospinal fluid lactate dehydrogenase levels in early stroke and transient ischemic attacks," Stroke, vol. 21, no. 6, pp. $854-$ 857, 1990.

[17] M. Thoresen, X. Liu, S. Jary et al., "Lactate dehydrogenase in hypothermia-treated newborn infants with hypoxicischaemic encephalopathy," Acta Paediatrica, vol. 101, no. 10, pp. 1038-1044, 2012.

[18] H. Chu, C. Huang, J. Dong et al., "Lactate dehydrogenase predicts early hematoma expansion and poor outcomes in intracerebral hemorrhage patients," Translational Stroke Research, vol. 10, no. 6, pp. 620-629, 2019.

[19] L. Hansheng, C. Yuan, and X. Zongyi, "Relationship between serum cardiac troponin T level and outcome in insular lobe hemorrhage patients," Chinese Journal of Geriatric Heart Brain and Vessel Diseases, vol. 21, no. 3, pp. 286-289, 2019.

[20] K. Shibazaki, K. Kimura, K. Sakai, J. Aoki, and Y. Sakamoto, "Plasma brain natriuretic peptide is elevated in the acute phase of intracerebral hemorrhage," Journal of Clinical Neuroscience, vol. 21, no. 2, pp. 221-224, 2014.

[21] M. L. James, R. Blessing, B. G. Phillips-Bute, E. Bennett, and D. T. Laskowitz, "S100b and BNP predict functional neurological outcome after intracerebral hemorrhage," Biomarkers, vol. 14, no. 6, pp. 388-394, 2009.

[22] Y. Goya, K. Shibazaki, K. Sakai et al., "Brain natriuretic peptide upon admission as a biological marker of short-term mortality after intracerebral hemorrhage," European Neurology, vol. 71, no. 3-4, pp. 203-207, 2014.

[23] F. R. Marins, M. Limboro-Filho, B. F. D'Abreu et al., "Autonomic and cardiovascular consequences resulting from experimental hemorrhagic stroke in the left or right intermediate insular cortex in rats," Autonomic Neuroscience, vol. 227, article 102695, 2020.

[24] S. Oppenheimer and D. Cechetto, "The insular cortex and the regulation of cardiac function," Comprehensive Physiology, vol. 6, no. 2, pp. 1081-1133, 2016.

[25] F. R. Marins, M. Limborço-Filho, C. H. Xavier et al., "Functional topography of cardiovascular regulation along the rostrocaudal axis of the rat posterior insular cortex," Clinical and Experimental Pharmacology \& Physiology, vol. 43, no. 4, pp. 484-493, 2016.

[26] M. Sykora, T. Steiner, S. Poli, A. Rocco, P. Turcani, and J. Diedler, "Autonomic effects of intraventricular extension in intracerebral hemorrhage," Neurocritical Care, vol. 16, no. 1, pp. 102-108, 2012.

[27] C.-H. Chen, S.-C. Tang, D.-Y. Lee et al., "Impact of supratentorial cerebral hemorrhage on the complexity of heart rate variability in acute stroke," Scientific Reports, vol. 8, no. 1, p. 11473, 2018.

[28] S. De Raedt, A. De Vos, and J. De Keyser, “Autonomic dysfunction in acute ischemic stroke: an underexplored therapeutic area?," Journal of the Neurological Sciences, vol. 348, no. 1-2, pp. 24-34, 2015.

[29] M. Dutsch, M. Burger, C. Dorfler, S. Schwab, and M. J. Hilz, "Cardiovascular autonomic function in poststroke patients," Neurology, vol. 69, no. 24, pp. 2249-2255, 2007.

[30] V. Chakradhar and J. Kasal, "Cardiac dysfunction in adult patients with traumatic brain injury: a prospective cohort study," Clinical Medicine \& Research, vol. 16, no. 3-4, pp. 57-65, 2018.

[31] M. Estévez-Báez, C. Machado, B. García-Sánchez et al., “Autonomic impairment of patients in coma with different Glasgow 
Coma Scale (GCS) assessed with heart rate variability," Brain Injury, vol. 33, no. 4, pp. 496-516, 2019.

[32] F. C. David, "Cortical control of the autonomic nervous system," Experimental Physiology, vol. 2, no. 99, pp. 326-331, 2014.

[33] N. Kuriyama, T. Mizuno, F. Niwa, Y. Watanabe, and M. Nakagawa, "Autonomic nervous dysfunction during acute cerebral infarction," Neurological Research, vol. 32, no. 8, pp. 821-827, 2010.

[34] A. Racho, A. Briosa, L. Pereira, M. Rodrigues, and T. Geraldes, "Thalamic role in the autonomic nervous system: the evidence of a case of Takotsubo cardiomyopathy following thalamic ischemic stroke," Clinical Neurology and Neurosurgery, vol. 200, article 106378, 2021.

[35] N. Grassl, S. Baumann, M. Kruska et al., "Acute ischemic stroke and elevated troponin: Diagnostic work-up and therapeutic consequences," Deutsche Medizinische Wochenschrift, vol. 146, no. 8, pp. 534-541, 2021.

[36] S. Fu, R. Jin, L. Luo, and P. Ye, "Baseline type 2 diabetes had a significant association with elevated high sensitivity cardiac troponin T levels in Chinese community-dwelling population: a 5-year prospective analysis," Nutrition \& Metabolism, vol. 14, no. 1, p. 73, 2017.

[37] K. Sharain, V. C. Vasile, Y. Sandoval et al., "The elevated highsensitivity cardiac troponin T pilot: diagnoses and outcomes," Mayo Clinic Proceedings, vol. 96, no. 9, pp. 2366-2375, 2021.

[38] J. F. Scheitz, C. H. Nolte, U. Laufs, and M. Endres, “Application and interpretation of high-sensitivity cardiac troponin assays in patients with acute ischemic stroke," Stroke, vol. 46, no. 4, pp. 1132-1140, 2015.

[39] C. H. Huang, W. J. Chen, W. T. Chang, P. K. Yip, and Y. T. Lee, "QTc dispersion as a prognostic factor in intracerebral hemorrhage," American Journal of Emergency Medicine, vol. 22, no. 3, pp. 141-144, 2004.

[40] A. Hays and M. N. Diringer, "Elevated troponin levels are associated with higher mortality following intracerebral hemorrhage," Neurology, vol. 66, no. 9, pp. 1330-1334, 2006.

[41] R. Sandhu, W. S. Aronow, A. Rajdev et al., "Relation of cardiac troponin I levels with in-hospital mortality in patients with ischemic stroke, intracerebral hemorrhage, and subarachnoid hemorrhage," American Journal of Cardiology, vol. 102, no. 5, pp. 632-634, 2008.

[42] B. V. Maramattom, E. M. Manno, J. R. Fulgham, A. S. Jaffe, and E. F. Wijdicks, "Clinical importance of cardiac troponin release and cardiac abnormalities in patients with supratentorial cerebral hemorrhages," Mayo Clinic Proceedings, vol. 81, no. 2, pp. 192-196, 2006. 